\title{
Crecimiento de árboles individuales de Geoffreea decorticans Burk., en un bosque del Chaco semiárido, Argentina
}

\section{Growth of individual trees of Geoffreea decorticans Burk. in a forest from the Semiarid Chaco, Argentina}

\author{
Ana María Giménez ${ }^{1 *}$, Patricia Hernández ${ }^{1}$, \\ Norfol Ríos ${ }^{1}$ y Federico Calatayu ${ }^{1}$
}

\begin{abstract}
RESUMEN
El objetivo del trabajo es contribuir al conocimiento del crecimiento de árboles individuales de Geoffreea decorticans (chañar), definir en función de la edad la evolución del incremento radial (IR), diámetro a la altura del pecho (DAP), sección normal (SN) y estimar el incremento medio (IMA) y anual (IA) con el fin proponer directrices para la correcta gestión de la especie. El estudio fue realizado en un bosque del Chaco Semiárido, Parque Los Quebrachos, Alberdi, Santiago del Estero, Argentina. Para el análisis epidométrico de fuste se derribaron 16 individuos que cumplían con la condición de: DAP superior a $15 \mathrm{~cm}$; fuste sin defectos; de buena vitalidad. De los árboles derribados se extrajeron rodajas de $5 \mathrm{~cm}$ de espesor, a las alturas de 0,30 m; 1,30 m y final del fuste. La marcación y medición de anillos se efectuó con el Equipo Computarizado ANIOL y el programa CATRAS. Los datos fueron analizados con el software estadístico IFOSTAT. El chañar presenta anillos de crecimiento bien demarcados. El incremento radial promedio es $5,90 \mathrm{~mm}(1,79-16,55)$. La edad de culminación del crecimiento en DAP es a los 5 años y en SN se logra a partir de los 37 años. Este crecimiento permite definir el manejo en rotaciones cortas.
\end{abstract}

PALABRAS CLAVE:

Anillos de crecimiento, chañar, crecimiento radial, edad del árbol, manejo forestal.

\begin{abstract}
The objective of this study was to improve the knowledge on the annual growth rates of Geoffreea decorticans (chañar), to define the evolution of annual growth increment (IR), diameter at breast height (DAP), normal section (DN) with age, to estimate mean annual (IMA) and annual increment (IA) in order to propose guidelines for proper management of the species. The study was carried out in a semiarid Chaco forest, at Quebrachos Park, Alberdi, Santiago del Estero Province, Argentina. For epidometric stem analysis, 16 vigorous trees were selected based on DAP greater than $15 \mathrm{~cm}$ and clean bole. From each tree cross-sections of $5 \mathrm{~cm}$ thickness were removed at $0,30 \mathrm{~m} ; 1,30 \mathrm{~m}$ above ground, and at the end of the stem. Definition of ring boundaries and ring with measuring were made with computerized equipment ANIOL and the computer program CATRAS. The data were analyzed with the statistical software package INFOSTAT. Chañar presented well-defined annual growth rings. The average thickness of rings is $5,90 \mathrm{~mm}(1,79-16,55)$. The age of completion for DAP was determined to be the five
\end{abstract}

1 Instituto de Silvicultura y Manejo de Bosques (INSIMA). Facultad de Ciencias Forestales. Universidad Nacional de Santiago del Estero (UNSE). Argentina.

Autor para correspondencia. C.e.: amig@unse.edu.ar 
years, and NS is achieved after 37 years. This growth rate can define the management on short rotations.

KEY WORDS:

Growth rings, chañar, radial growth, age of tree, forest management.

\section{INTRODUCCIÓN}

Las especies arbóreas del género Geoffroea que vegetan en Argentina son: Geoffroea striata Morong (manduvi-rá) y Geoffroea decorticans Burkart (chañar). Ambas tienen importancia florística y son usadas localmente, ya que sus maderas tienen escaso valor comercial. La madera y la corteza de esta especie han sido descritos en forma comparada y se consideran de alta afinidad (Giménez, 2004). El chañar es una especie arbórea secundaria de amplia distribución geográfica, se extiende desde el sur de Perú, norte de Chile, Bolivia, Chaco Paraguayo y oeste de Uruguay, hasta la Patagonia Argentina. En este último país ocupa principalmente las provincias fitogeográficas del Chaco, Monte y Espinal, desde Jujuy a Río Negro y Neuquén (Coscaron Arias y Ganmdullo, 2004). Puede encontrarse como un integrante secundario del bosque chaqueño, o formando rodales puros en zonas abiertas, inundables, con niveles freáticos poco profundos y salinos. Es una especie colonizadora de suelos húmedos, ya consolidados y ricos en limos, muy agresiva en el Chaco Boreal (Mereles y Degen, 1997). En el Chaco semiárido es una de las especies de mayor frecuencia, presente en $80 \%$ de las áreas de estudio junto a Cercidium praecox, Prosopis nigra y Prosopis alba (Giménez y Hernández, 2008).

El chañar se reproduce por semillas - por vía vegetativa mediante raíces gemíferas y cepa. Las semillas poseen diseminación endozoica. La activación de yemas en las ramas, corona del tallo o las raíces pueden ser provocadas, entre otros factores, por el fuego. Es alta la capacidad de rebrote después de sufrir incendios y cortes severos.

Esta especie tiene dos aspectos a considerar, en algunas zonas de Argentina es reconocida como una leñosa arbustiva invasora; mientras que en bosques heterogéneos del Chaco semiárido es un componente secundario. Es apreciada localmente por el uso de su madera y otros valores (Giménez y Moglia, 2003). En las zonas semiáridas y subhúmedas forma densas colonias, debido a que su sistema radical da origen a un conjunto de plantas dispuesta en forma circular u oval, llamadas isletas, con plantas de mayor tamaño y edad, próximas al centro. En algunas regiones, se relaciona a la especie con perjuicios que ocasiona al comportarse como invasora en pastizales degradados, sin considerar los importantes productos que ofrece (Anderson, 1976). La concepción que la biomasa de chañar puede tener uso industrial, no ha sido debidamente desarrollada, con aspectos positivos complementarios, obteniendo ventajas productivas (ganadera y forestal) económicas y ecológicas. En la implementación de sistemas silvopastoriles debe tomarse en cuenta la cobertura y la densidad de individuos, regulando mediante podas y raleos la entrada de luz y la circulación del ganado (Echeverría y Giulietti, 2001).

Es de interés regional, investigar sobre el crecimiento y ecología del chañar con el fin de darle un adecuado manejo. Una herramienta válida para tal análisis es el estudio de crecimiento a partir del conteo y medición de anillos. El ancho de los anillos de crecimiento es el parámetro que tradicionalmente se emplea para inferir cambios en la historia de vida de los árboles y la relación con su entorno. El empleo de los anillos de crecimiento de las leñosas permite cuantificar en forma precisa 
las velocidades de crecimiento radial de los árboles en distintos ambientes, así como determinar diferencias en las estructuras poblacionales del bosque en relación con factores tales como el clima, el suelo, los disturbios naturales y la historia de uso (Villalba, 2000). Estudios que requieran información sobre la estructura de edad y la dinámica de los bosques, la determinación de sus turnos de aprovechamiento, el establecimiento de las relaciones entre crecimiento leñoso, o simplemente la reacción del bosque a un tratamiento silvícola determinado, necesitan del fechado y la evaluación de los ritmos pasados y presentes del crecimiento (Schweingruber, 1996).

El conocimiento de la estructura y dinámica de estos bosques permitirá plantear estrategias de manejo y recuperación de los mismos (Villagra et al., 2002). La aplicación del análisis epidométrico en el manejo es directa y representa una herramienta indispensable en la planificación de aprovechamientos sustentables de bosques. Las tasas de crecimiento observadas en las diferentes especies nativas estudiadas, indican que los turnos biológicos de corta son más largos de lo que se ha pensado y se viene aplicando hasta la actualidad (Giménez y Ríos, 1999; Giménez et al., 2000; Giménez et al., 2003). La modelación del crecimiento del leño de especies nativas en su ambiente, permite la estimación de los momentos en que los árboles alcanzan el máximo crecimiento medio, información muy útil para una apropiada corta, asegurando la renovación del monte (Juárez de Galíndez y Balzarini, 2003).

\section{OBJETIVOS}

El objetivo del presente trabajo es contribuir al conocimiento del crecimiento de árboles individuales de Geoffreea decorticans; definir la evolución del incremento radial (IR), diámetro a la altura del pecho (DAP), sección normal (SN) con la edad, estimar el incremento medio (IMA) y el incremento anual (IA), evaluando las perspectivas de la especie para un futuro manejo sustentable.

\section{METODOLOGÍA}

El estudio fue realizado en el Parque Los Quebrachos, Departamento Alberdi, Provincia de Santiago del Estero, Argentina. El área corresponde al Distrito Chaqueño Occidental y su posición geográfica es 2604'19,62" Lat. S.; 6335'41,40" Long. O (Fig. 1). El clima es subtropical, continental, de carácter semiárido, con precipitaciones escasas y estivales alternando con sequías en períodos bien delimitados. La precipitación media anual es de $600 \mathrm{~mm}$ (para un intervalo de $500 \mathrm{~mm}$ a $700 \mathrm{~mm}$ ).

El área de estudio se localiza a escasos metros al este de la llanura de escurrimiento impedido del río Salado (bañados activos). Su localización geomorfológica coincide con la antigua llanura de inundación, la cual fue cubierta por un manto superficial de sedimentos eólicos. Como consecuencia el material originario de los suelos se halla íntimamente relacionado al comportamiento del río Salado y a la posterior cobertura superficial. Los suelos son planos, suavemente elevados con vegetación boscosa (Piarfon, 2005).

El predio en estudio es un bosque secundario de dos quebrachos, clausurado hace 20 años, lo que permitió a la vegetación expresarse con gran exuberancia (Giménez y Hernández, 2008). La estructura vertical diferencia 4 estratos: 1) herbáceo y gramíneo, de cobertura completa con predominio de Trichloris crinita; Solanum argentinum, Heliotropium sp., Setaria fiebrigi; Bromelia hierony; 2) arbustivo, de $3 \mathrm{~m}$ de altura compuesto por Celtis pallida, Acacia precoz; 3) arbó- 
República Argentina

Provincia de Santiago del Estero

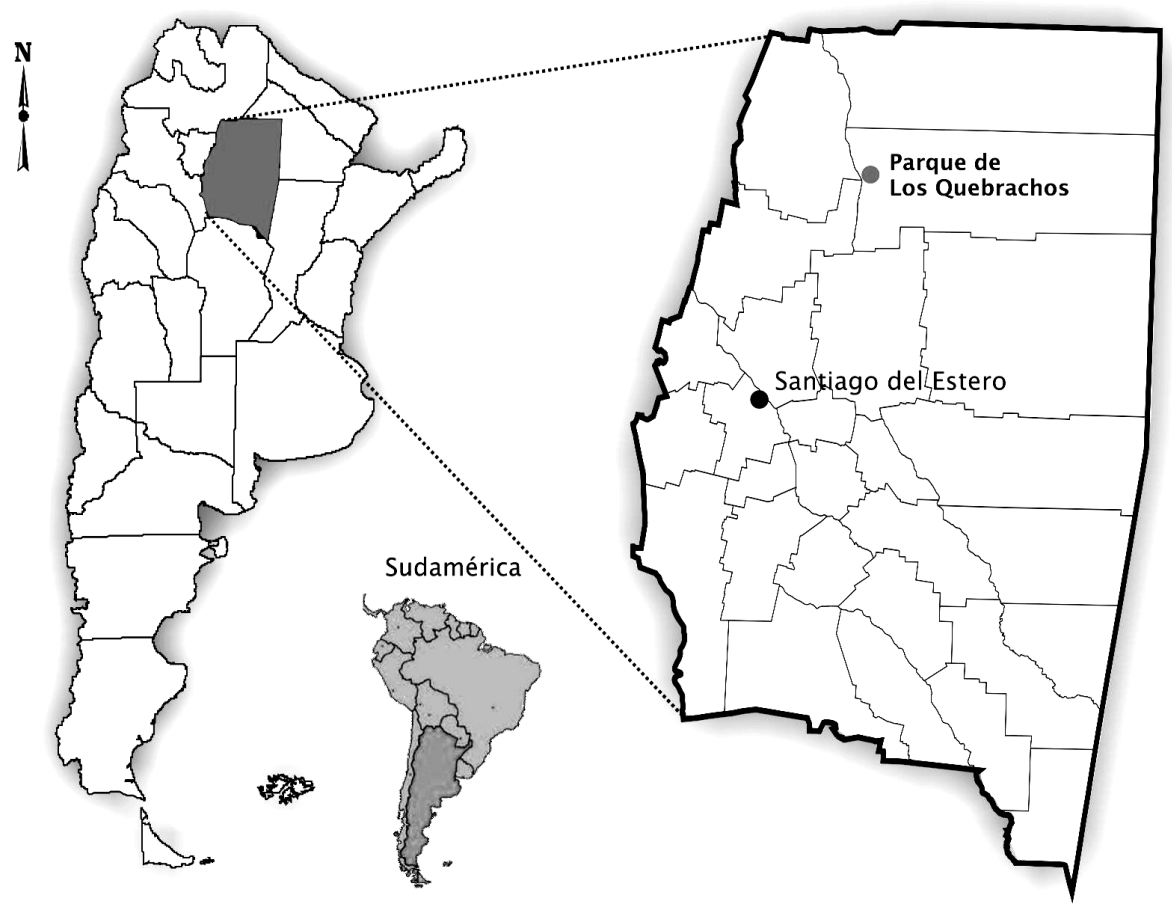

Figura 1. Área de estudio.

reo inferior, formado por especies secundarias: Ziziphus mistol, Prosopis ruscifolia, Cercidium praecox, Prosopis nigra, Geoffroea decorticans (con alturas de $6 \mathrm{~m}$ a 8 m); 4) arbóreo superior formado por Schinopsis lorenzii (quebracho colorado) $y$ Aspidosperma quebracho-blanco (quebracho blanco) con alturas de $12 \mathrm{~m}$ a 20 $\mathrm{m}$. Las leñosas más abundantes son: Schinopsis lorenzii (44\%), Zizipphus mistol, Celtis tala, Prosopis nigra, Geoffroea decorticans (10\%). Según datos del Inventario forestal, el chañar está representado en el rodal con 28 árboles/ha, volumen de fuste de $3,335 \mathrm{~m}^{3} / \mathrm{ha}$ y volumen total de $6,11 \mathrm{~m}^{3} /$ ha (Piarfon, 2005).

El chañar está distribuido en pequeños grupos. Es una especie de cuarta magnitud, corteza verdosa y flores amarillas muy llamativas (Fig. 2). (Giménez y Mogia, 2003). La distribución por clases diamétricas indica un rodal joven, con escasos pies de diámetros superiores a 20 $\mathrm{cm}$, en clases de edad entre 5 y 20 años, con predominio de la clase diamétrica de 5 $\mathrm{cm}$ a $10 \mathrm{~cm}$. Para el análisis epidométrico de fuste, se derribaron 16 individuos de la especie Geoffreea decorticans (Gill., ex Hook.\& Arn.) Burk., Familia: Fabaceae (Leguminosae: Papilionoideae).

De una muestra de árboles de diferentes edades tomada al azar, se escogieron los que cumplían la condición de: DAP superior a $15 \mathrm{~cm}$; fuste sin defectos; de buena vitalidad. A cada árbol seleccionado se midió el DAP a 1,30 m; altura de fuste (m) 

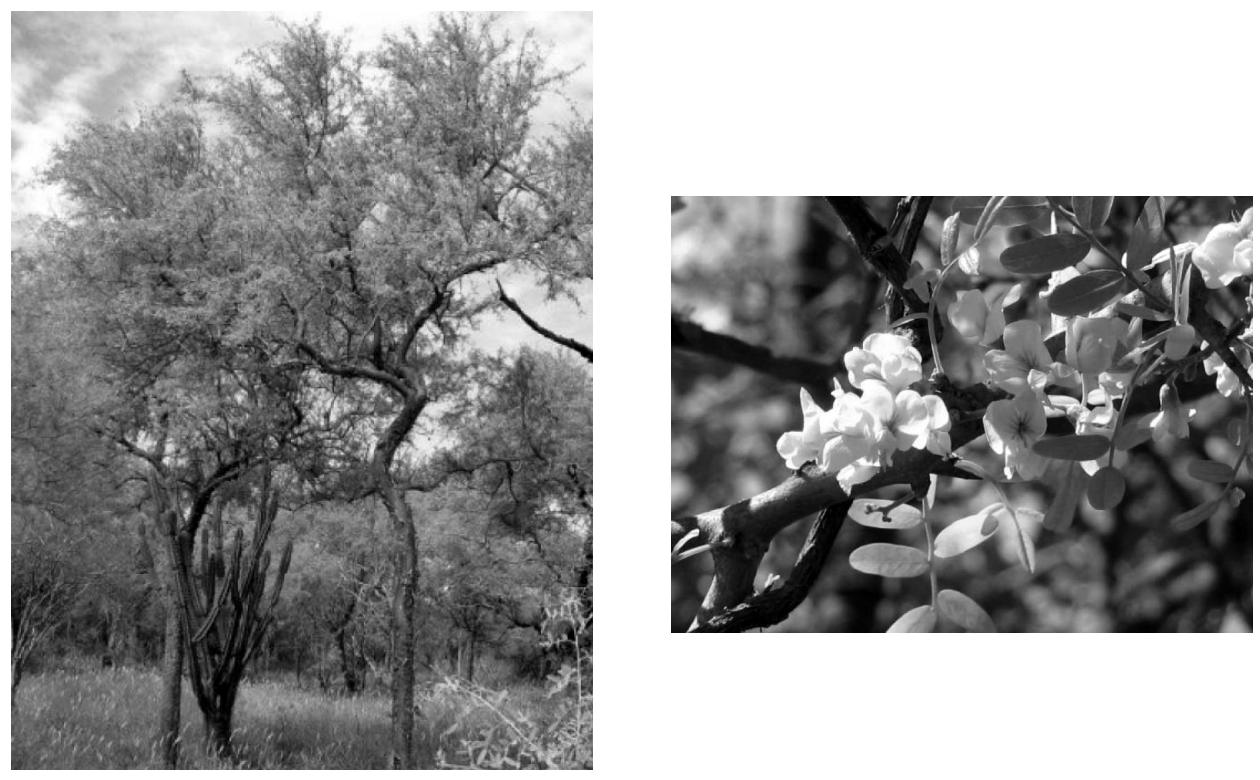

Figura 2. Detalles dendrológicos del chañar.

y altura total $(m)$, marcándose la dirección norte en el fuste. De los árboles derribados se extrajeron secciones trasversales de 5 $\mathrm{cm}$ de espesor a una altura sobre el suelo de $0,30 \mathrm{~m} ; 1,30 \mathrm{~m}$ y al final del fuste, acorde con metodología de Helinska (1991). Las muestras se prepararon con cepilladora (cepillo), lijadora de banda y orbital con juego de lijas de granulometría de 600 granos por pulgada cuadrada a 1000 granos por pulgada cuadrada, hasta tener una superficie lisa, que demarcara con alta definición los anillos de crecimiento.

La marcación y medición de anillos se efectuó con el Equipo Computarizado ANIOL y el programa CATRAS (Aniol, 1991). Con este procedimiento se consignó el número de años y el espesor del anillo con una resolución de 0,01 mm. Las mediciones se efectuaron de corteza a médula. Se sincronizaron los anillos manualmente y por cuadrantes, a fin de tener diferencia de edad ( \pm 2 años).
Se analizaron las siguientes variables en función de la edad:

IR: Incremento radial o espesor de anillo de crecimiento

DAP: Diámetro a la altura de 1,30 m

$\mathrm{SN}$ : Sección normal del árbol (sección a la altura de 1,30 m).

Se calculó el crecimiento medio anual (IMA) y crecimiento anual (IA) para las variables DAP y SN. El crecimiento medio anual (IMA) resulta del cociente entre el valor total acumulado de la variable considerada, en un determinado tiempo de su evolución, desde su origen y su edad en dicho momento. Incremento anual (IA) es la expresión del crecimiento anual que resulta del crecimiento en un año. El crecimiento anual y el crecimiento medio anual coinciden en un determinado momento de la vida del árbol o de la masa, en el cual el crecimiento medio anual es máximo. La edad de culminación biológica se obtuvo a 
partir del punto de encuentro de la curva del IMA y del IA.

$$
\begin{gathered}
\mathrm{IMA}=\text { Variable }(\mathrm{n}) / \mathrm{n} \\
\mathrm{IA}=\mathrm{Vn}-\mathrm{V}(\mathrm{n}-1)
\end{gathered}
$$

donde:

IMA: incremento medio anual

(n): año

IA: Incremento anual

$\checkmark n$ : variable en el año $n$

$V(n-1)$ : variable en el año $n-1$

\section{Análisis estadístico}

Los datos fueron analizados con el software estadístico programa IFOSTAT 2010. Para el estudio de las tendencias en crecimiento se usó el método basado en la edad biológica de los árboles (Komin, 1967), trabajándose con individuos de edades diferentes. Se empleó la técnica de suavizado de las series individuales de ancho de anillos para eliminar la variación climática de las series de IR, previa modelación del crecimiento. Se usó un filtro basado en medias móviles de ventana igual a 5 años (Juárez de Galíndez et al., 2007).

El espesor de los anillos de cada uno de ellos, se alineó con los de otros individuos según la edad biológica. Así se expresaron biológicamente las tendencias de crecimiento de la especie en determinado sitio. La estandarización de la curva de crecimiento medio presume que la forma de la estructura a cualquier edad biológica es independiente del período de tiempo durante el cual se produce.

Fueron analizados los modelos de regresión para las propiedades estudiadas y la edad. Se trabajó con un modelo polinomial de segundo orden para modelar la estructura de la media (promedio) de la variable IR en función de la edad del árbol.

Modelo de crecimiento:

$$
\mathrm{y}=\mathrm{B}_{0}+\mathrm{B}_{1^{*}} \mathrm{Edad}+\mathrm{B}_{2}{ }^{*} \mathrm{Edad}^{2}+\mathrm{E}_{0}
$$

\section{RESULTADOS}

El chañar es una de las pocas especies arbóreas del Chaco argentino que presenta anillos de crecimiento bien definidos, lo que permite realizar el análisis epidométrico del fuste por conteo y medición de anillos. A nivel macroscópico se demarca una banda oscura correspondiente a la sección de madera tardía, donde el tejido es más denso, seguida de una banda ancha y clara de madera temprana al inicio del anillo (Fig. 3).

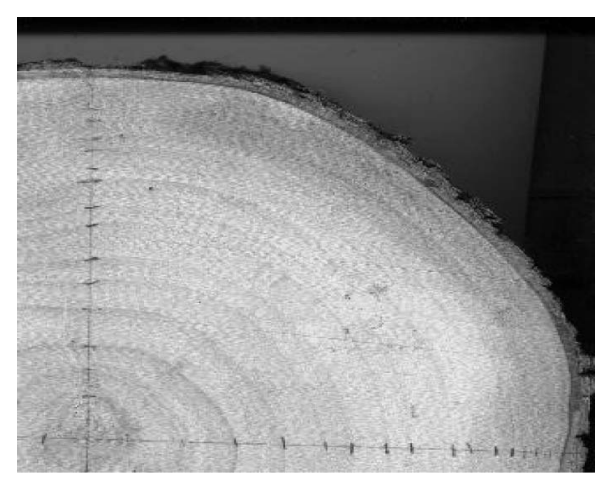

Figura 3. Vista macroscópica de los de anillos de chañar 


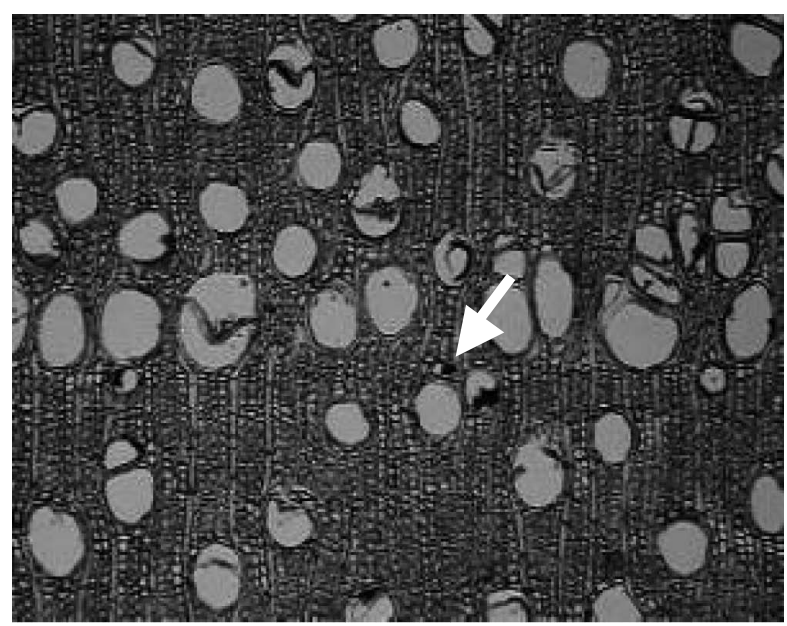

Figura 4. Vista microscópica del inicio de un anillo de chañar.

La definición del anillo está dada por la conjunción de dos aspectos anatómicos: la presencia de una banda de parénquima terminal, más la distribución de los vasos a lo largo del anillo en porosidad semicircular (Giménez, 2004) (Fig. 4).

En la tabla 1 se indican los estadísticos descriptivos de las series promedio de IR de 16 individuos. El espesor medio de anillos (IR) fue de 5,90 mm, con una intervalo de $1,79 \mathrm{~mm}$ a $16,55 \mathrm{~mm}$, desviación estándar de 2,54 y el coeficiente de variabilidad de $43 \%$. Los árboles estudiados mostraron edades que oscilan entre 9 a 33 años.

Los valores IR fueron variables entre los individuos de un mismo rodal (Fig. 5) con un valor máximo a los 5 años de edad.

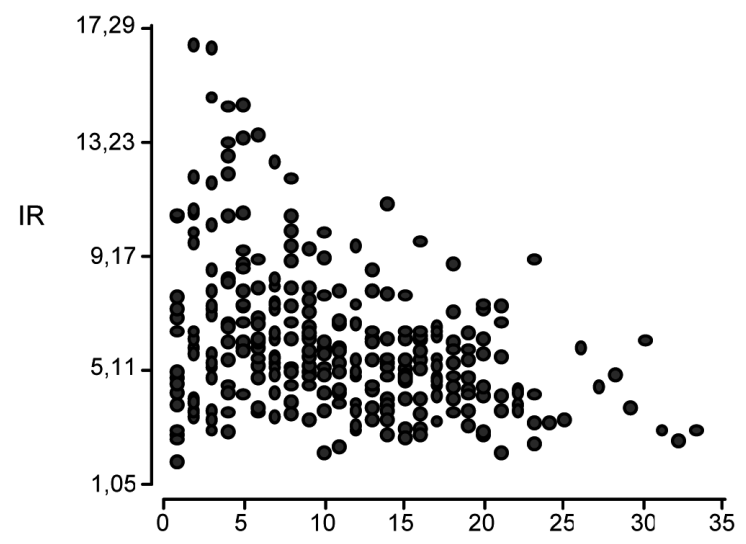

Figura 5. Relación entre las series IR y edad para el chañar. 
Tabla 1. Estadística Descriptiva de las series de IR de 16 árboles de chañar.

\begin{tabular}{|c|c|c|c|c|c|}
\hline IR & $\begin{array}{l}\text { Edad del árbol } \\
\text { (años) }\end{array}$ & $\begin{array}{c}\text { Media } \\
(\mathrm{mm})\end{array}$ & $\begin{array}{l}\text { Desviación } \\
\text { estándar } \\
\text { (mm) }\end{array}$ & $\begin{array}{l}\text { Valor mínimo } \\
\qquad(\mathrm{mm})\end{array}$ & $\begin{array}{c}\text { Valor máximo } \\
\qquad(\mathrm{mm})\end{array}$ \\
\hline Árbol 1 & 23 & 5,05 & 2,31 & 2,13 & 10,93 \\
\hline Árbol 2 & 19 & 5,23 & 1,63 & 2,84 & 8,88 \\
\hline Árbol 3 & 20 & 4,87 & 1,27 & 2,63 & 7,30 \\
\hline Árbol 4 & 33 & 4,87 & 1,56 & 2,49 & 8,99 \\
\hline Árbol 5 & 17 & 9,75 & 3,35 & 5,06 & 16,52 \\
\hline Árbol 6 & 15 & 7,45 & 3,2 & 2,97 & 12,61 \\
\hline Árbol 7 & 21 & 6,27 & 1,55 & 4,06 & 10,47 \\
\hline Árbol 8 & 16 & 6,13 & 2,27 & 1,79 & 10,49 \\
\hline Árbol 9 & 16 & 5,3 & 2,05 & 2,77 & 9,50 \\
\hline Árbol 10 & 29 & 5,14 & 1,47 & 2,52 & 7,82 \\
\hline Árbol 11 & 21 & 4,45 & 2,05 & 2,07 & 10,19 \\
\hline Árbol 12 & 10 & 11,09 & 3,49 & 6,89 & 16,55 \\
\hline Árbol 13 & 10 & 7,48 & 1,95 & 4,15 & 10,63 \\
\hline Árbol 14 & 9 & 8,57 & 2,27 & 4,48 & 11,98 \\
\hline Árbol 15 & 26 & 5,43 & 1,84 & 2,72 & 9,77 \\
\hline Árbol 16 & 20 & 5,73 & 1,52 & 2,81 & 9,06 \\
\hline
\end{tabular}

En la figura 6 se indica el histograma de frecuencia relativa de IR.

En la tabla 2 se indican las funciones de ajuste de las variables IR, DAP y SN

Dada la variabilidad del $\mathrm{IR}$, las series se suavizaron con medias móviles en escala de 5 años. Se ajustaron a una curva explicada por una función polinómica de segundo grado con un $\mathrm{R}^{2}$ de 0,51 (Fig.7).

La evolución DAP/edad (Fig. 8) se ajustó a una ecuación polinómica de segundo grado con un $\mathrm{R}^{2}$ de $0,84(30 \mathrm{~cm}$ DAP a los 31 años). 


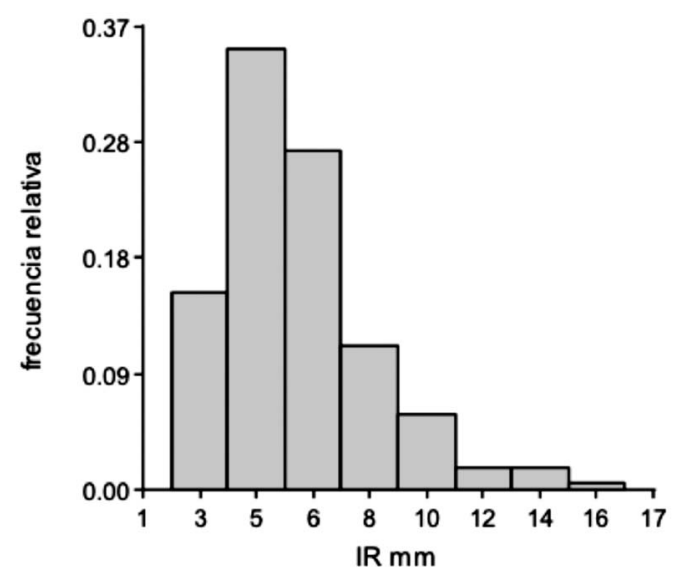

Figura 6. Frecuencia relativa (\%) del IR (mm).

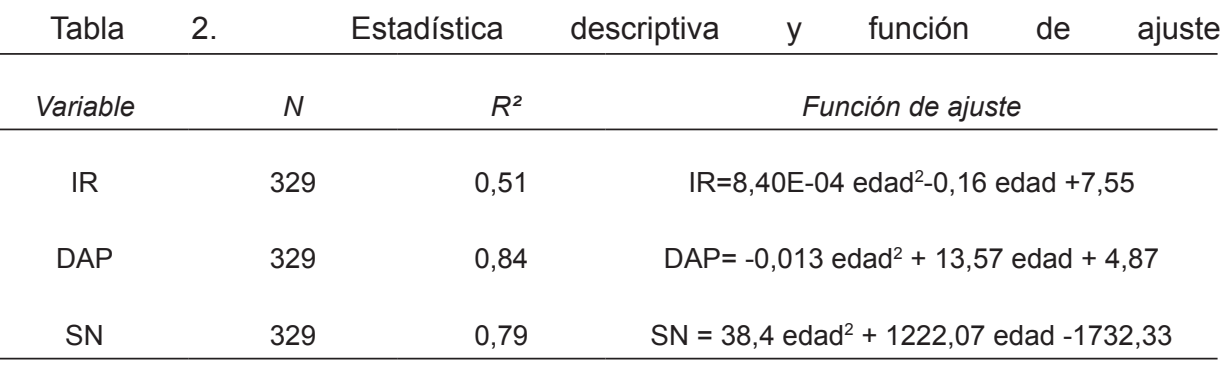

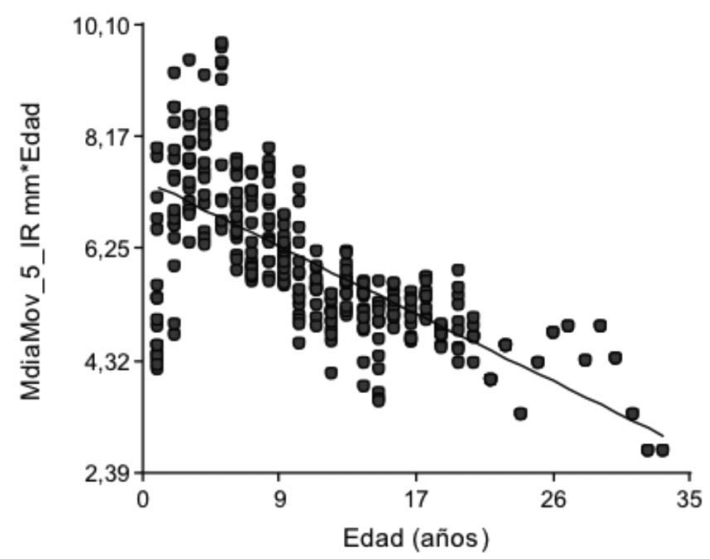

Figura 7. Ajuste del IR suavizado. 
El incremento promedio en diámetro para un intervalo de 33 años fue de 0,879 $\mathrm{cm}$. Este dato es excepcional en las leñosas arbóreas del Chaco Semiárido, ya que se alcanza el diámetro mínimo de corta a tan temprana edad. Se estimó el IMA e IA en función del DAP (Fig. 9), a los 33 años; el IMA es de $9,16 \mathrm{~mm}$ y IA de 5,82 $\mathrm{mm}$. Se encontró que el IMA e IA se interponen tempranamente a los 5 años a un valor de $11,81 \mathrm{~mm}$.

La sección normal en relación con la edad se ajustó a una función polinómica de segundo grado con $\mathrm{R}^{2}$ de 0,79 (Fig. 10).

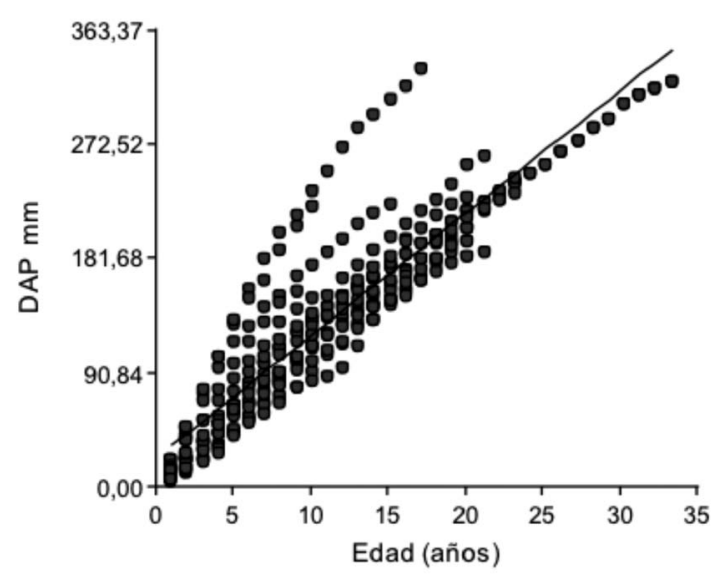

Figura 8. Evolución del DAP y la edad.

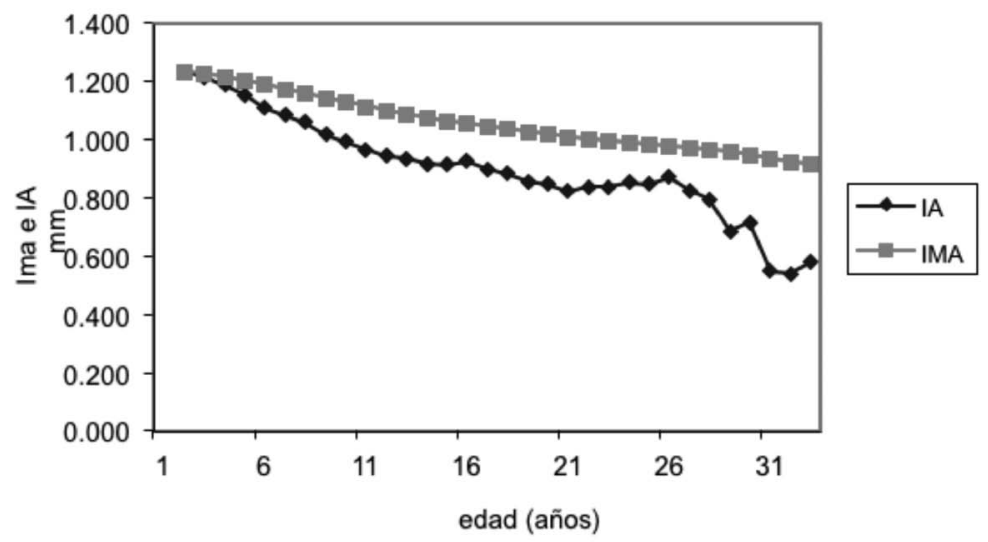

Figura 9. Culminación del incremento en DAP según IMA e IA. 
Se obtuvieron valores calculados y ajustados de IMA, $2738 \mathrm{mmm}$, y de IA, $2176 \mathrm{~mm}^{2}$, a la edad de 33 años (Fig. 11). Las curvas están prontas a interceptarse, lo que indica una culminación temprana del crecimiento expresado en sección, con máximos entre los 20 y 25 años.
Para definir el turno biológico de corta de una población, se determinó la edad de la planta donde el crecimiento anual (crecimiento promedio en una edad determinada) es menor al crecimiento anual promedio alcanzado hasta ese momento (crecimiento acumulado dividido por la edad de la planta).

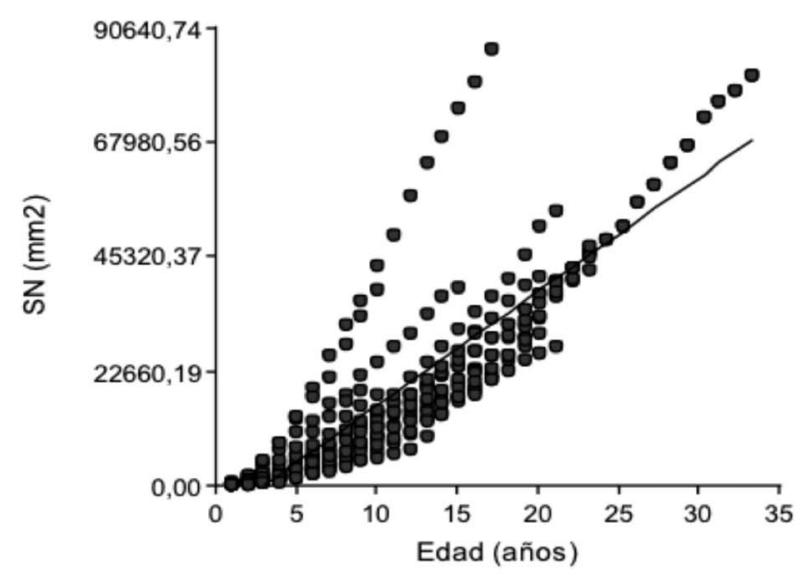

Fig. 10. Evolución de SN y edad.

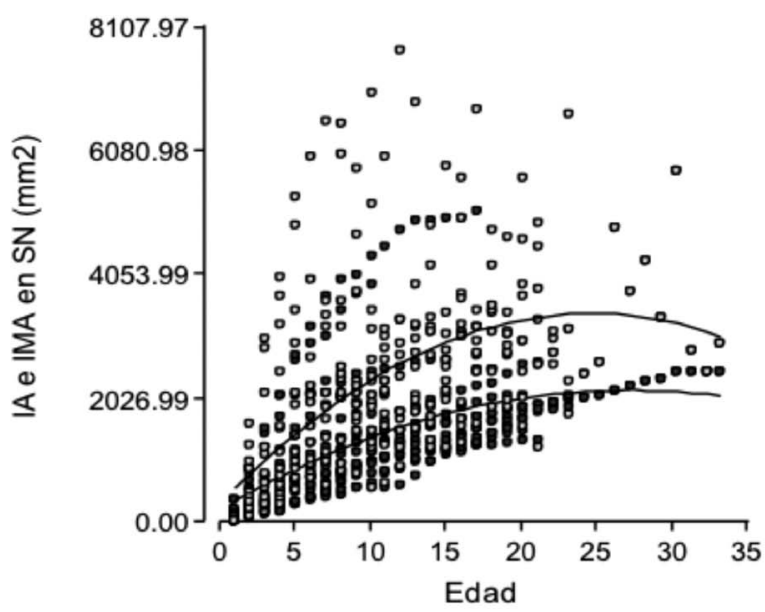

Figura 11. IMA e IA en función de la SN. 


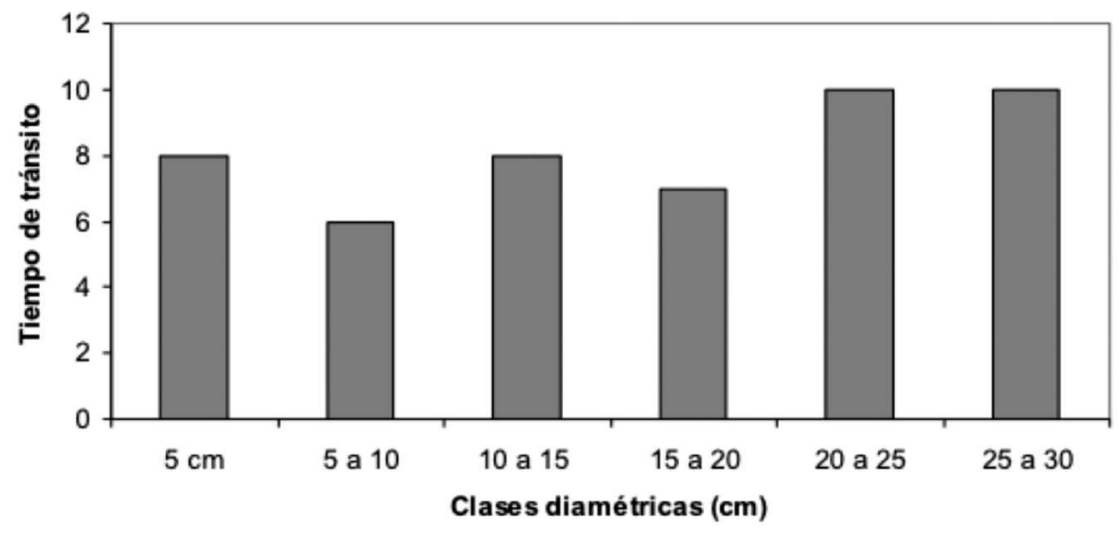

Figura 12. Tiempo de tránsito por clases diamétricas.

Como la variable que mejor se correlaciona con el volumen económico es la sección normal (SN), se estimó el incremento anual en SN para cada edad a partir del incremento diamétrico medido. Con la ecuación de ajuste, se proyectó la evolución de la SN a través de sus incrementos, lográndose interceptar las curvas de IMA e IA a la edad de 37 años para un valor de $2240 \mathrm{~mm}^{2}$. Esto permitió especular sobre los diámetros máximos citados por la literatura y el punto de culminación del crecimiento en SN. La edad de culminación expresada en SN fue de 37 años en el caso de este estudio. Se alcanzó el diámetro mínimo de corta a los 25 años.

Con la curva de DAP y edad se estimó el tiempo de tránsito entre cada clase diamétrica de $5 \mathrm{~cm}$, que varió entre 6 a 10 años según las clases (Fig.12).

\section{DISCUSIÓN}

Existe una literatura rica en relación con estudios del espesor medio de los anillos de chañar. Roth y Giménez (2006) indican un valor de $3,1 \mathrm{~mm}$ (1 $\mathrm{mm}$ a $5 \mathrm{~mm}$ ) para el Chaco Semiárido. Echeverría y Giulietti (2001) citan un espesor medio de $3,4 \mathrm{~mm} /$ año para San Luis, con crecimiento en diámetro de $0,65 \mathrm{~cm} / a n ̃ o$. En chañarales de Chile, Serra (1998) revela un crecimiento en diámetro de $0,7 \mathrm{~cm}$. En este estudio el espesor medio de anillos para 33 años de intervalo fue de 5,90 $\mathrm{mm} / \mathrm{año}$.Se notó una marcada diferencia en la velocidad de crecimiento con la edad. El crecimiento fue muy rápido durante los primeros 5 años de vida y alcanzó un máximo entre los 2 a 5 años (5,9 mm) para decrecer a los 30 años, lo que indicó la culminación temprana de crecimiento.

Juárez de Galíndez y Balzarini (2003), trabajando con especies nativas, sugieren la técnica de suavizado aplicada a las series de anchos de anillos para eliminar la variación climática de las series individuales, previa modelación del crecimiento. Los árboles objeto de estudio indicaron un comportamiento de una especie de rápido crecimiento y longevidad baja para las condiciones ecológicas del Chaco. Este resultado implica la necesidad de replantear la mayoría de los emprendimientos forestales existentes, incluso la legislación forestal. Tradicionalmente se ha aconsejado cortar árboles mayores a $25 \mathrm{~cm}$ de diámetro para espe- 
cies secundarias, esto significa cortar árboles antes de que alcancen la mayor tasa de crecimiento forestal. El diámetro mínimo de corta se logra a los 25 años.

En referencia a los anillos de crecimiento, las características de demarcación y el espesor varía según las especies de la región. En Prosopis alba el espesor medio de anillos es $4,05 \mathrm{~mm}(2,11 \mathrm{~mm}$ a 5,18 mm) (Giménez et al., 1998); Prosopis kuntzei, 3,26 mm (1,9 $\mathrm{mm}$ a 4,5 mm) (Giménez et al., 1997); Prosopis nigra, $3,29 \mathrm{~mm}(1,14 \mathrm{~mm}$ a $8,59 \mathrm{~mm}$ ) (Giménez et al., 2003); Prosopis ruscifolia, 5,66 mm (3,3 a 6,8 mm) (Giménez et al., 2009); Schinopsis quebracho-colorado, 2,19 mm (1,6 mm a 4,5 mm) (Giménez y Ríos, 1999) y Aspidosperma quebracho-blanco con 2,20 mm (Moglia, 2000).Juárez et al. (2007) indican para vinal (Prosopis ruscifolia) una edad de culminación del incremento radial a los 11 años de edad, mientras que para la especie de este estudio fue de cinco años.

Como se evidencia, Geoffroea decorticans es la especie de mayor IR promedio de las estudiadas para el Chaco Semiárido, lo cual representa una aliciente muy importante. Hay que destacar que el diámetro máximo de $30 \mathrm{~cm}$, citado por Tortorelli (2009), es indicativo de la baja longevidad de la especie.

\section{CONCLUSIONES}

Geoffreea decorticans es una especie de crecimiento medio y de muy buenas perspectivas de aprovechamiento en relación con el tiempo de tránsito de las clases diamétricas.El Incremento radial promedio (IR) es de 5,90 mm (1,79 mm -16,55 mm), con un máximo a edades tempranas (5 años), para un intervalo de 35 años.La edad de culminación expresada en DAP fue a los 5 años, en SN se logra a partir de los 37 años; el diámetro mínimo de corta se logra a los 25 años. Este crecimiento permite el manejo en rotaciones cortas.

\section{REFERENCIAS}

Anderson, D. 1976. Invasión del chañar (Geoffroea decorticans) en los pastizales de la Pcia de San Luis. RIA (7):153-172.

Aniol, R. 1991. Computer Aided Tree Rings Analysis System, User manual. Schleswig, F.R.G, Alemania.

Gandullo, R., J. Gastiazoro, A. Bünzli y C. Coscaron-Arias. 2004. Flora típica de las bardas del Neuquén y sus alrededores. Petrobrás. 246p.

Echeverría, J.C. y J. Giulietti. 2001. Estimación de biomasa y productividad de chañar mediante teledetección y modelos de simulación. INTA Ria. 30:59-66.

Giménez, A.; N. Ríos, y G. Moglia. 1997. Leño y corteza de Prosopis kuntzei en relación a algunas magnitudes dedrométricas. Revista de Investigaciones Agraria Sistemas y Recursos Forestales- España- 6(1-2):163-182.

Giménez, A.; N. Ríos.; G. Moglia y C. López. 1998. Leño y corteza de Prosopis alba Griseb., algarrobo blanco, en relación con algunas magnitudes dendrométricas. Bosque 19(2):53-62.

Giménez, A.M. y N.A. Ríos. 1999. Crecimiento de Schinopsis quebrachocolorado (Schlecht.) Barkl. et Meyer, Anacardiaceae. Madera y Bosques 5(2):35-51.

Giménez, A.; G. Moglia; P. Hernández y S. Bravo. 2000. Leño y corteza de Prosopis nigra (Griseb.) Hieron, Mimosaceae, en relación a algunas 
magnitudes dendrométricas. Revista Forestal Venezolana 44(2):29-37.

Giménez, A.M. y J.G. Moglia. 2003. Árboles del Chaco Argentino. Guía para el reconocimiento dendrológico. ISBN: 987 95852-9-1. Ed. Secretaria de Ambiente y Desarrollo Sustentable. Ministerio de Desarrollo Social Facultad de Ciencias Forestales. Universidad Nacional de Santiago del Estero. 307 p.

Giménez, A.M., N.A. Ríos y J.G. Moglia. 2003. Crecimiento de Prosopis nigra (Griseb.) Hieron (Algarrobo negro) en Santiago del Estero, Argentina. Foresta Veracruzana 5(2):17-21.

Giménez, A.M. 2004. Anatomía comparada de leño y corteza de Geoffroea striata y Geoffroea decorticans. Madera y Bosques 10(1):55- 68.

Giménez, A.M. y P. Hernández. 2008. Biodiversidad en Ambientes naturales del chaco Argentino Vegetación del Chaco semiárido, Provincia de Santiago del Estero Fascículo 1-FONCYT. FCF-UNSE. 120 p. ISBN: 978-987-137526-4.

Giménez, A.M.; N. Ríos; P. Hernández y J.G. Moglia. 2009. Influencia de la edad en crecimiento de vinal (Prosopis ruscifolia Burkart.) en la provincia de Santiago del Estero. Madera y Bosques 15(2):45-54.

Juárez de Galíndez, M. y M. Balzarini. 2003. Modelación de la estructura de covarianza entre observaciones de un mismo individuo en modelos de curvas de crecimiento arbóreo. SAE 17(1-2) (on line).

Juárez de Galíndez, Giménez, A.; Ríos, N.; M. Balzarini, 2007. Modelación del crecimiento en diámetro de vinal
(Prosopis ruscifolia) en Santiago del Estero, Argentina. Foresta Veracruzana 9(2):9-15.

Komin, G. 1967. Methods of calculation of growth indices for trees of different age in totality. Unpublished research report . Citado por Stokes y. Smiley, 1968.

Mereles F. y R. Degen. 1997. Leñosas colonizadoras e indicadoras de sitios modificados en el Chaco Boreal, Paraguay. Rojasiana 4(1):25-83.

Moglia, J.G. 2000. Variabilidad radial de los caracteres anatómicos de Aspidosperma quebracho blanco Schelkt Apocinaceae. Tesis doctoral, Universidad Nacional de Tucumán, Argentina. $95 p$.

PIARFON. 2005. Parque Chaqueño, Subregión Semiárida Estudio de los sistemas productivos en montes nativos explotados en el Parque Chaqueño Subregión Semiárido. Universidad Nacional de Santiago del Estero (UNSE). Tomo I, 240 p.

Roth, I. y A.M. Giménez. 2006. Argentine Chaco forests. Dendrology, tree structure, ang economic use. 2- The humid Chaco. Encyclopedia of plant anatomy.XIV/5. Gerbruder-Borntraeger-Berlin-Stuttgart. 204 p.

Schweingruber, F.H. 1996. Tree rings and environment: Dendroecology. Paul Haupt Berna, Suiza. 609 p.

Serra, M. 1998. Especies arbóreas y arbustivas para las zonas áridas y semiáridas de América Latina. Geoffreea decorticans. FAO. Red Latinoamericana de Cooperación Técnica en Sistemas Agroforestales. Web: http//www.rlc.fao.org/redes/sisag/ arboles/Chi-geof.htm. 
Tortorelli, L. 2009. Maderas y Bosques argentinos. $2^{\mathrm{a}}$ ed. Tomo I y II. Orientación Gráfica Editora. 576 p.

Villagra, P.; M. Morales; R. Villalba y J. Boninsegna. 2002. Dendroecología de los algarrobales de la zona árida argentina. IANIGLA. Mendoza, Argentina. $\mathrm{p}: 53-57$.

Villalba, R. 2000. Dendroclimatology: a southern hemisphere perspective. In:
Smolka P.P. y W. Volkheimer, eds. Southern hemisphere paleo- and neoclimates: Springer Verlag. Berlin, Alemania. p:27-57.

Helinska, R., E. Raczkowska; G. Fabisiak. 1991. Radial variation and growth rate in the length of the axial elements of sessile oak wood. IAWA Bull. 12(3):257-262. ticans Burk. en un bosque del Chaco semiárido, Argentina. Madera y Bosques 19(1):37-51. 